\title{
Tricuspid-valve regurgitant jet velocity as a risk factor for death in $\beta$-thalassemia
}

Pulmonary hypertension $(\mathrm{PH})$ has been recognized as a frequent complication in patients with $\beta$-thalassemia, owing to the underlying chronic anemia, endothelial damage, and hypercoagulable state., ${ }^{1,2}$ However, there are mounting concerns that reported prevalence rates using echocardiographic thresholds overestimate the true epidemiology of the disease. In 2014, we published results from a large multicenter study of 1,309 Italian $\beta$-thalassemia patients which evaluated $\mathrm{PH}$ prevalence through a dedicated protocol for right heart catherization (RHC), as part of the Italian Webthal ${ }^{\circledR}$ project (clinicaltrials gov. Identifier: NCT01496963). ${ }^{3}$ In the original study, adult ( $\geq 18$ years) patients with a diagnosis of $\beta$-thalassemia major or intermedia and without chronic restrictive lung disease or a left ventricular ejection fraction (LVEF) $\leq 50 \%$ were recruited between January 2012 and January 2013. Patients first underwent a screening transthoracic echocardiography using continuous-wave Doppler sampling of the peak tricuspid-valve regurgitant jet velocity (TRV) to calculate systolic pulmonary artery pressure (SPAP), based on which they were divided into three groups: PH unlikely $(n=1,234)$, SPAP $\leq 36 \mathrm{~mm}$ Hg or TRV $\leq 3.0 \mathrm{~m} / \mathrm{s}$; PH possible $(n=28)$, sPAP $>36$ to $<40 \mathrm{~mm} \mathrm{Hg}$ or TRV $>3.0$ to $<3.2 \mathrm{~m} / \mathrm{s}$; and PH likely $(n=47)$, sPAP $\geq 40 \mathrm{~mm}$ $\mathrm{Hg}$ or $\mathrm{TRV} \geq 3.2 \mathrm{~m} / \mathrm{s}$. Patients with $\mathrm{PH}$ likely underwent $\mathrm{RHC}$ and the prevalence of confirmed $\mathrm{PH}$ in the entire study population was $2.1 \%$. The positive predictive value for the TRV $\geq 3.2 \mathrm{~m} / \mathrm{s}$ threshold for the diagnosis of $\mathrm{PH}$ was $93.9 \% .3$ This latter finding was adopted by $\beta$-thalassemia international management guidelines which now recommend $\mathrm{RHC}$ in patients with a TRV $\geq 3.2 \mathrm{~m} / \mathrm{s}^{2,4}$

In this study, we provide long-term mortality data for patients who had a TRV $\geq 3.2 \mathrm{~m} / \mathrm{s}$ in the original study to further confirm the clinical value of this threshold to flag patients who require $\mathrm{RHC}$ and $\mathrm{PH}$-directed management. The Institutional Review Boards of all participating centers approved the study protocol and all participants signed a written informed consent before inclusion in the study. We followed 42 of the 47 patients with TRV $\geq 3.2$ $\mathrm{m} / \mathrm{s}(\mathrm{PH}$ likely) at the initial echocardiographic assessment (baseline) until March 2021, death, or loss to follow-up. Five patients had transitioned care to other

Table 1. Comparison of study parameters between baseline tricuspid-valve regurgitant jet velocity groups.

\begin{tabular}{|c|c|c|c|}
\hline \multirow[b]{2}{*}{ Parameter } & \multicolumn{2}{|c|}{ Baseline TRV } & \multirow[t]{2}{*}{ P-value* } \\
\hline & $\begin{array}{l}\geq 3.2 \mathrm{~m} / \mathrm{s} \\
(\mathrm{N}=42)\end{array}$ & $\begin{array}{c}>3.0 \text { to }<3.2 \mathrm{~m} / \mathrm{s} \\
(\mathrm{N}=26)\end{array}$ & \\
\hline \multicolumn{4}{|l|}{ Baseline characteristics } \\
\hline Age in years, median (IQR) & $48(39.8-57.8)$ & $44.5(39.3-48.3)$ & 0.097 \\
\hline Male, \% & 38.1 & 46.2 & 0.614 \\
\hline Splenectomized, \% & 88.1 & 76.9 & 0.312 \\
\hline $\begin{array}{l}\text { Thalassemia diagnosis, \% } \\
\beta \text {-thalassemia intermedia } \\
\beta \text {-thalassemia major }\end{array}$ & $\begin{array}{l}66.7 \\
33.3\end{array}$ & $\begin{array}{l}50 \\
50\end{array}$ & 0.208 \\
\hline Hemoglobin in $\mathrm{g} / \mathrm{dL}$, median (IQR) & $9.1(8-9.6)$ & $9.1(8.5-9.7)$ & 0.495 \\
\hline Serum ferritin in $\mathrm{ng} / \mathrm{mL}$, median (IQR) & $970(476.8-1,538.5)$ & $767.5(500-1,097)$ & 0.466 \\
\hline LVEF in \%, median (IQR) & $60(60-66.5)$ & $62(53.5-66)$ & 0.843 \\
\hline TRV in $\mathrm{m} / \mathrm{s}$, median (IQR) & $3.43(3.31-4.09)$ & $3.04(3.04-3.08)$ & $<0.001$ \\
\hline \multicolumn{4}{|l|}{ Management } \\
\hline $\begin{array}{l}\text { PH-directed therapy, } \%^{\dagger} \\
\text { None } \\
\text { Single agent } \\
\text { Double agent }\end{array}$ & $\begin{array}{l}35.7 \\
42.9 \\
21.4\end{array}$ & $\begin{array}{c}100 \\
0 \\
0\end{array}$ & $<0.001$ \\
\hline \multicolumn{4}{|l|}{ Outcome } \\
\hline Death from any cause, \% & 47.6 & 15.4 & 0.009 \\
\hline Death from cardiopulmonary disease, \% & 33.3 & 0 & 0.001 \\
\hline
\end{tabular}

*Comparisons made using the Mann Whitney $U$ test for continuous variables and Fisher's Exact test for categorical variables. †Bosentan, ambrisentan, sildenafil, tadalafil, macitentan, riociguat, and other angiotensin converting enzyme inhibitors, calcium channel and betablockers, and anticoagulants. IQR: interquartile range; LVEF: left ventricular ejection fraction; TRV: tricuspid-valve regurgitant jet velocity; PH: pulmonary hypertension. 
institutions immediately after the original study and were not included in this analysis. For comparisons, we also followed 26 of the 28 patients ( 2 patients transitioned care) originally grouped as $\mathrm{PH}$ possible (TRV $>3.0$ to $<3.2$ $\mathrm{m} / \mathrm{s}$ ). For each patient, we retrieved data on baseline demographics (age and sex), thalassemia diagnosis, splenectomy status, hemoglobin and serum ferritin levels (mean over previous 10 years), and LVEF. The use of $\mathrm{PH}-$ directed therapies during the period of observation and last observation TRV values, if available, were also retrieved.

Patients with a baseline TRV $\geq 3.2 \mathrm{~m} / \mathrm{s}(\mathrm{n}=42)$ and TRV $>3.0$ to $<3.2 \mathrm{~m} / \mathrm{s}(\mathrm{n}=26)$ had comparable age, hemoglobin level, serum ferritin level, and LVEF (Table 1). They also had comparable proportions of males, $\beta$-thalassemia intermedia diagnosis, and splenectomy (Table 1). None of the patients with a TRV $>3.0$ to $<3.2 \mathrm{~m} / \mathrm{s}$ received $\mathrm{PH}-$ directed therapy, while 18 (42.9\%) patients received single

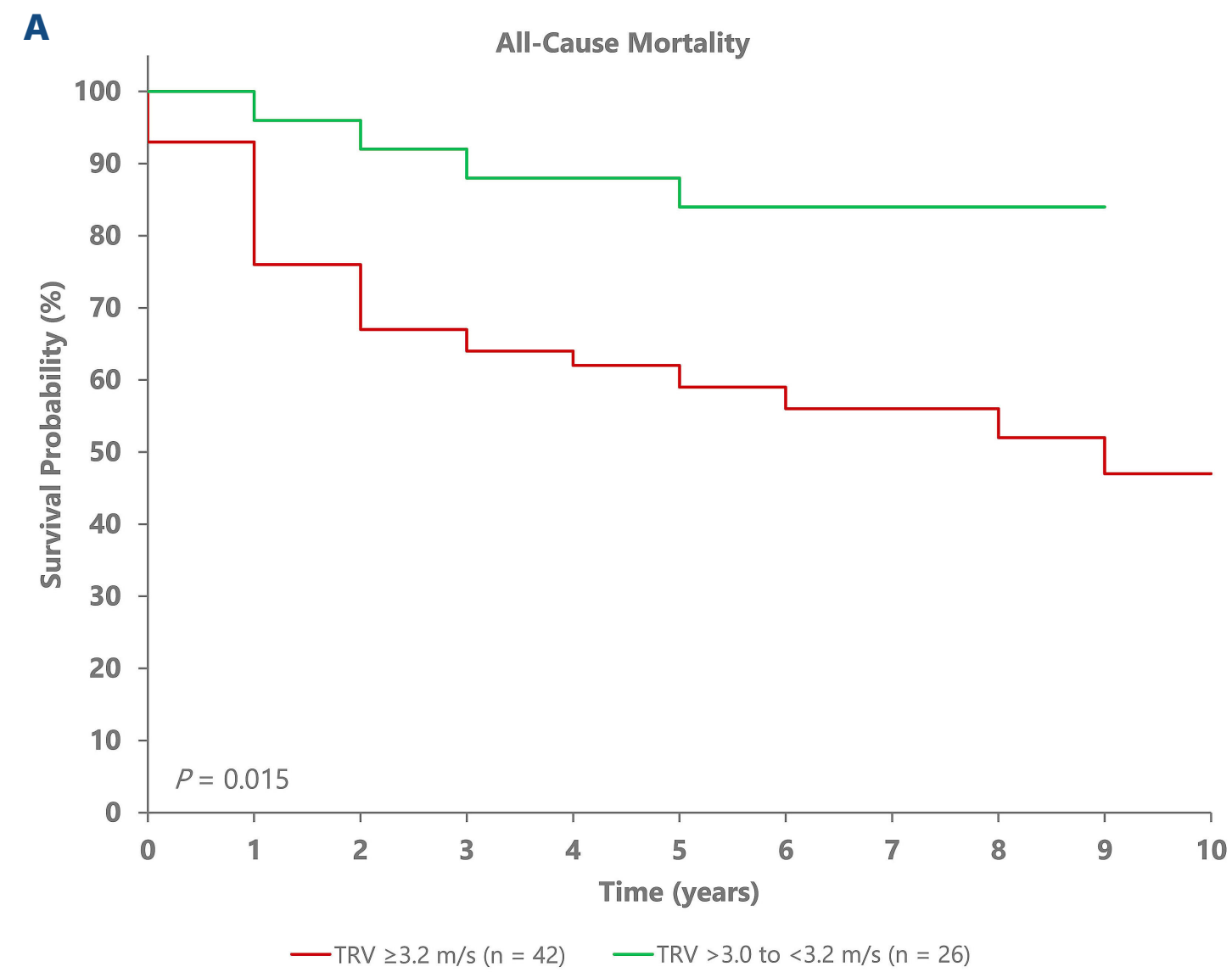

B

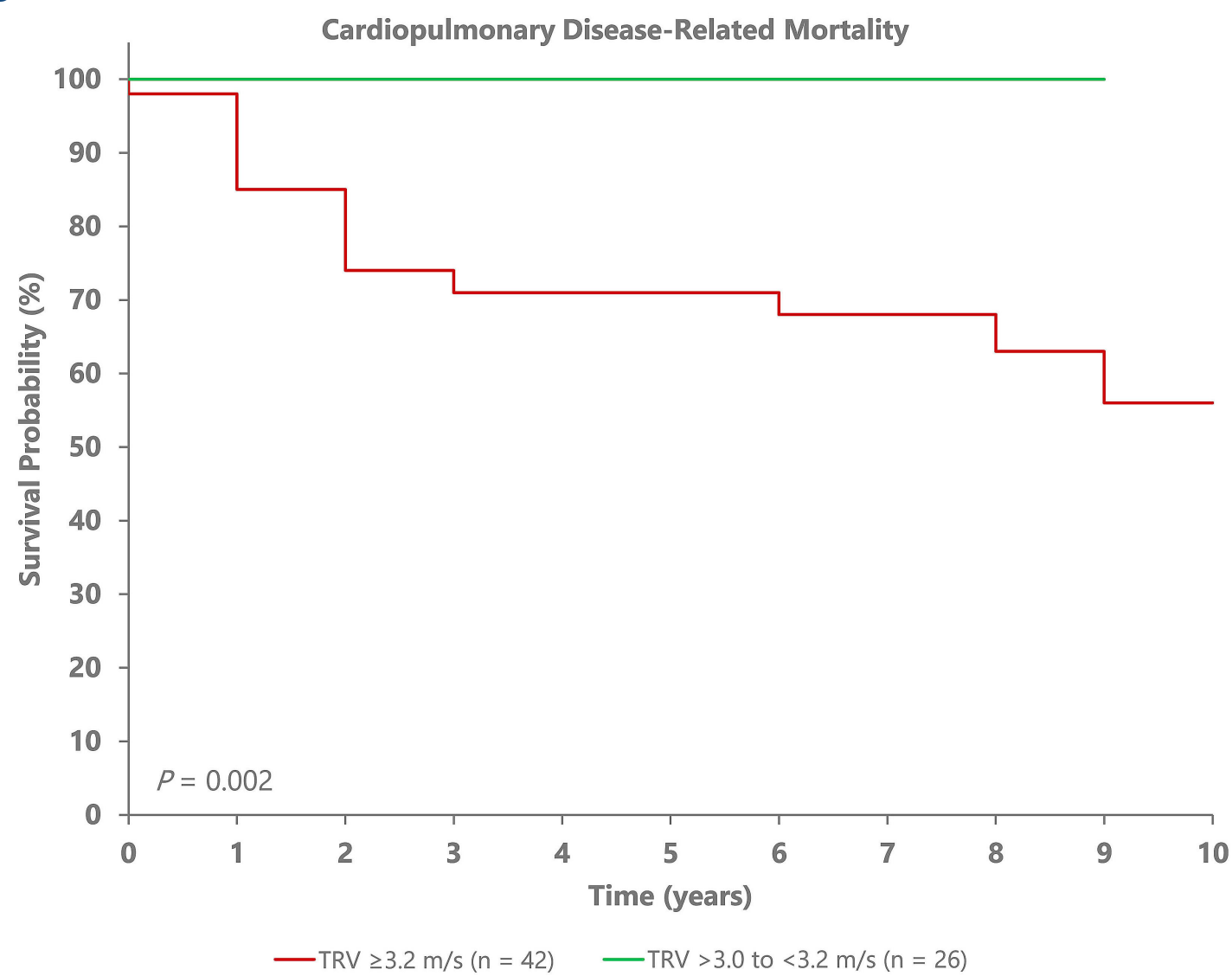

Figure 1. Kaplan-Meier survival curves. (A) All-cause mortality, and (B) cardiopulmonary disease-related mortality according to baseline TRV group. TRV: tricuspid-valve regurgitant jet velocity. 
A

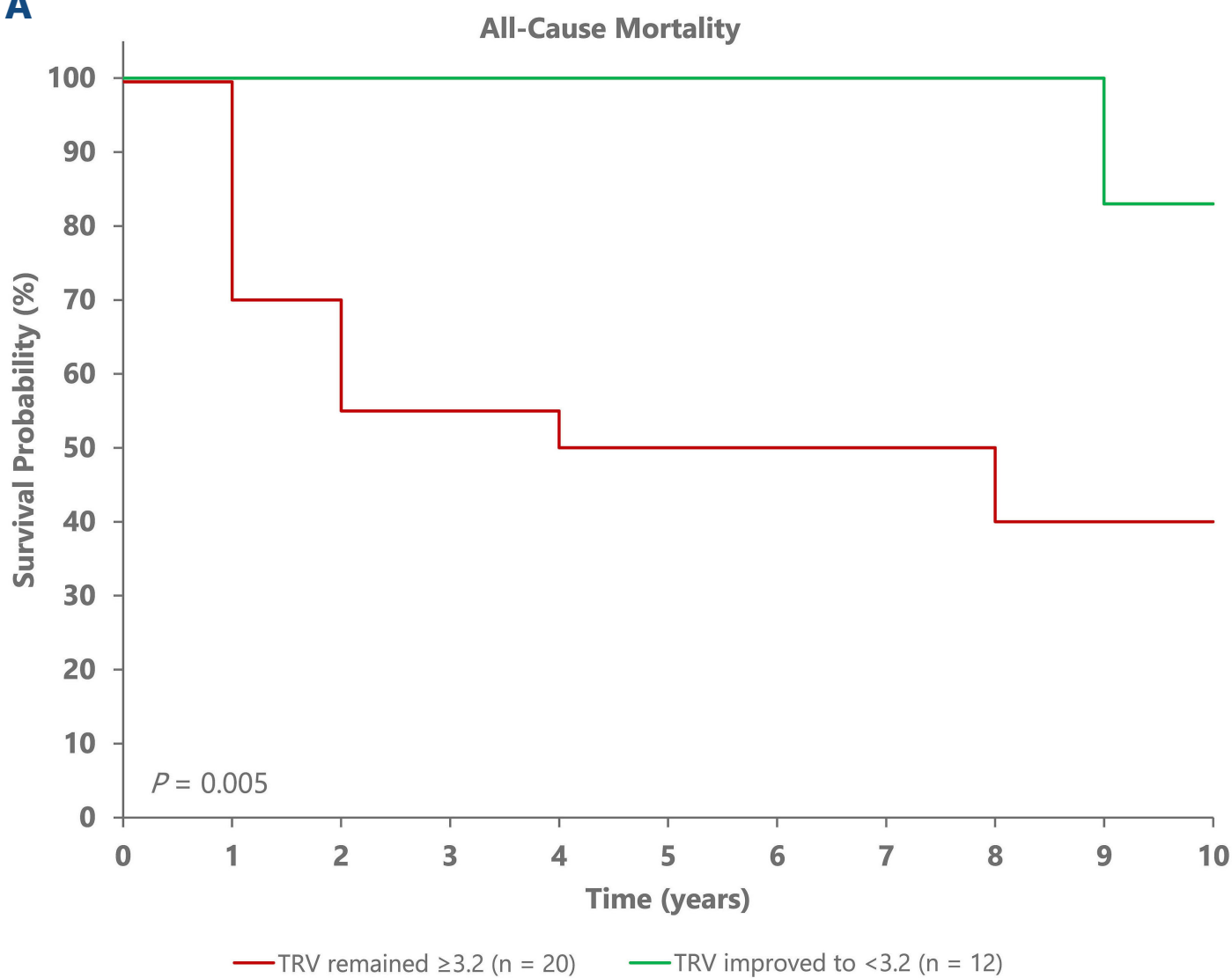

B

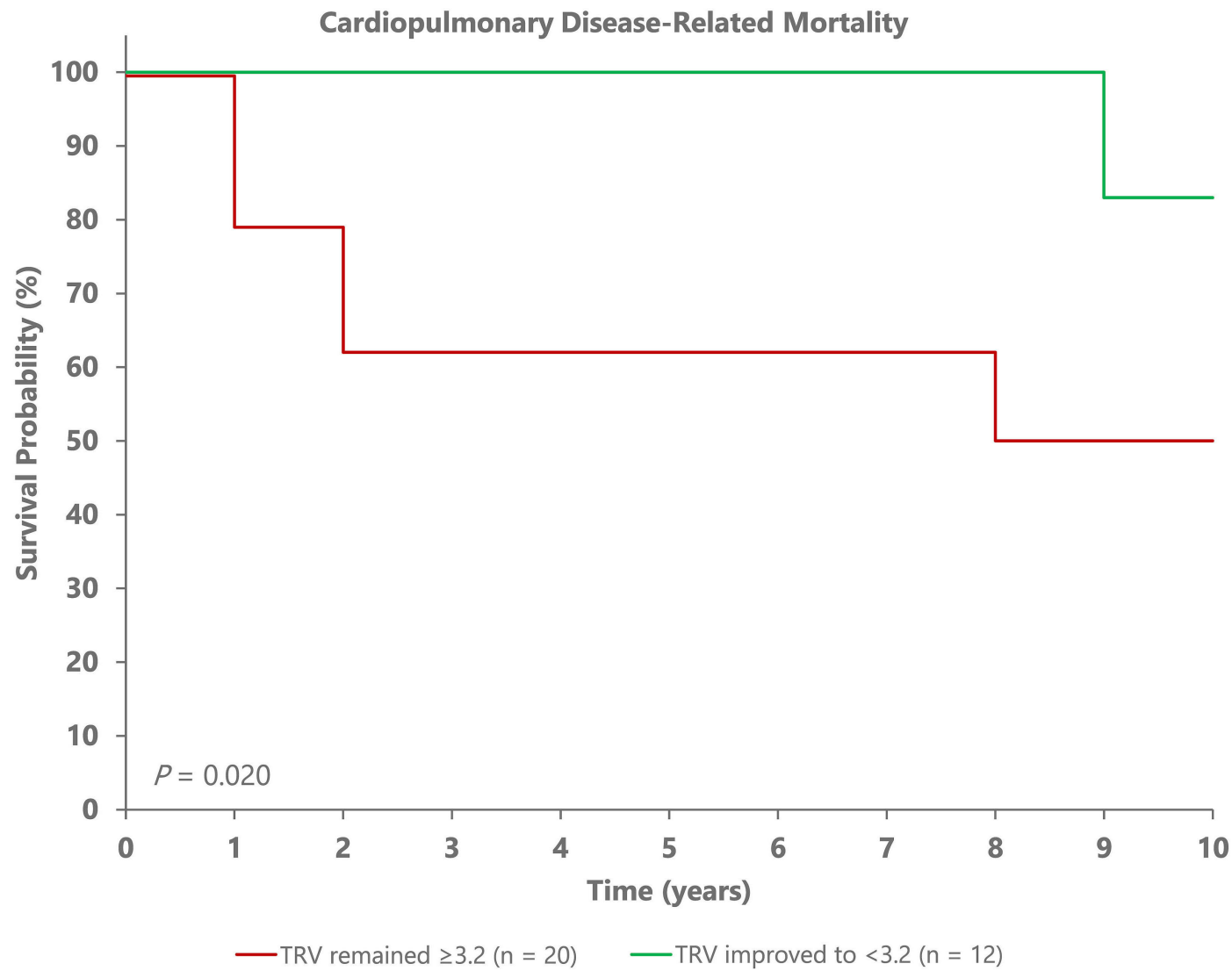

Figure 2. Kaplan-Meier survival curves. (A) All-cause mortality, and (B) cardiopulmonary disease-related mortality according to TRV improvement. TRV: tricuspid-valve regurgitant jet velocity.

agent and nine (21.4\%) patients received double agent therapy in the TRV $\geq 3.2 \mathrm{~m} / \mathrm{s}$ group (Table 1 ).

The median follow-up time was 6 years (interquartile range [IQR]: 2.3-8). The crude all-cause mortality rate was 47.6\% (95\% confidence interval [CI]: 32.0-63.6; cardiopulmonary disease $n=14$, hepatic failure $n=2$, post fracture $n=1$, severe hemolytic anemia $n=1$, unknown $n=2$ ) in patients with a baseline TRV $\geq 3.2 \mathrm{~m} / \mathrm{s}$ compared with 15.4\% (95\% Cl: 4.4-34.9; hepatic failure $n=2$, sepsis $n=1$, unknown = 1) in patients with a TRV $>3.0$ to $<3.2 \mathrm{~m} / \mathrm{s}$ $(P=0.009$; Table 1). The crude cardiopulmonary diseaserelated mortality rate was 33.3\% (95\% Cl: 19.6-49.6; right-sided heart failure $n=12$, pulmonary embolism $n=2$ ) in patients with a baseline TRV $\geq 3.2 \mathrm{~m} / \mathrm{s}$ while there were no cardiopulmonary disease-related deaths in patients with a TRV $>3.0$ to $<3.2 \mathrm{~m} / \mathrm{s}(P=0.001$; Table 1$)$. On KaplanMeier curves, cumulative all-cause mortality-free survival estimates at 5 and 9 years for patients with a baseline 
TRV $\geq 3.2 \mathrm{~m} / \mathrm{s}$ were $59 \%$ and $47 \%$ compared with $84 \%$ and $84 \%$ in patients with a TRV $>3.0$ to $<3.2 \mathrm{~m} / \mathrm{s}$ (log-rank chi-square: 5.944, $P=0.015$; Figure $1 \mathrm{~A}$ ). Cumulative cardiopulmonary disease-related mortality-free survival estimates at 5 and 9 years for patients with a baseline TRV $\geq 3.2 \mathrm{~m} / \mathrm{s}$ were $71 \%$ and $56 \%$ compared with $100 \%$ and $100 \%$ in patients with a TRV $>3.0$ to $<3.2 \mathrm{~m} / \mathrm{s}$ (log-rank chi-square: 9.413, $P=0.002$; Figure 1B). Of note, 29 of the 42 patients with a baseline TRV $\geq 3.2 \mathrm{~m} / \mathrm{s}$ underwent RHC in the original study, two patients did not have confirmed $\mathrm{PH}$ on RHC and remained alive; while 27 patients had confirmed $\mathrm{PH}$ on $\mathrm{RHC}$, out of which 15 died during the period of observation (12 due to cardiopulmonary disease).

On Cox regression analysis, the unadjusted hazard ratio (HR) for death from any cause in patients with a baseline TRV $\geq 3.2 \mathrm{~m} / \mathrm{s}$ compared with TRV $>3.0$ to $<3.2 \mathrm{~m} / \mathrm{s}$ was 3.402 (95\% Cl: 1.157-10.005; $P=0.026$ ). In a multivariate, forward stepwise Cox regression model including baseline TRV groups and adjusting for age, sex, splenectomy status, thalassemia diagnosis, hemoglobin level, serum ferritin level, LVEF, and $\mathrm{PH}$-directed therapy; only baseline TRV was significantly and independently associated with an increased risk of death (adjusted HR for TRV $\geq 3.2$ $\mathrm{m} / \mathrm{s}$ vs. TRV $>3.0$ to $<3.2 \mathrm{~m} / \mathrm{s}: 3.337$; $95 \% \mathrm{Cl}: 1.122-9.923$; $P=0.030$ ).

On receiver operating characteristic curve analysis, a baseline TRV of $3.2 \mathrm{~m} / \mathrm{s}$ had the highest sum of sensitivity and specificity for the outcomes of death from any cause (area under the curve $[A \cup C]: 0.67 \pm 0.07 ; P=0.024$ ) and death from cardiopulmonary disease (AUC: 0.74 \pm 0.07 ; $P=0.007$ ) among the range of TRV values $>3.0 \mathrm{~m} / \mathrm{s}$ included in this study.

Thirty-two out of the 42 patients with a baseline TRV $\geq 3.2$ $\mathrm{m} / \mathrm{s}$ had a TRV at the last observation. TRV values improved to $<3.2 \mathrm{~m} / \mathrm{s}$ in $12(37.5 \%)$ patients of whom three (25\%) were receiving single agent and five (41.7\%) were receiving double therapy. TRV values remained $\geq 3.2 \mathrm{~m} / \mathrm{s}$ in $20(62.5 \%)$ patients of which 14 (70\%) were receiving single agent and one (5\%) was receiving double therapy. Survival was significantly better in patients who had TRV improvement than those who did not for both all-cause mortality (log-rank chi-square: 7.876, $P=0.005$; Figure 2A) and cardiopulmonary disease-related mortality (log-rank chi-square: 5.237, $P=0.020$; Figure 2B).

Our study further highlights the relevance of a TRV $\geq 3.2$ $\mathrm{m} / \mathrm{s}$ threshold to inform $\mathrm{PH}$-related management in $\beta$ thalassemia by establishing an increased risk of mortality, especially from cardiopulmonary disease. Our original study supported a recommendation to undergo RHC at this TRV threshold through the finding of a high positive predictive rate, as well as more recent data on high mortality in those patients with confirmed $\mathrm{PH}$ on $\mathrm{RHC}^{2-5}$ However, not all patients were able to undergo RHC, which makes clinical correlates with adverse outcomes identified in the full subset of patients with a TRV $\geq 3.2$ $\mathrm{m} / \mathrm{s}$ in this study very informative. This may also support management decisions in resource-restricted settings or when RHC is not feasible. The key limitations in our work that need to be addressed in future studies include inability to evaluate lower TRV thresholds for long-term mortality outcomes or RHC correlation; however, the lack of cardiopulmonary disease-related deaths in the subset of patients with TRV $>3.0$ to $<3.2 \mathrm{~m} / \mathrm{s}$, further supports our original choice of a TRV $\geq 3.2 \mathrm{~m} / \mathrm{s}$ for further evaluation. Indeed, low mortality rates $(n=3$, heart failure, cancer, unknown) were previously reported in thalassemia cohorts with lower TRV thresholds ( $n=148$, mean TRV: $2.3 \pm 0.4 \mathrm{~m} / \mathrm{s}$, range: $0.2-3.5 ; 5 \%$ with $\mathrm{TRV} \geq 3.0 \mathrm{~m} / \mathrm{s}) .{ }^{6}$ On the contrary, data in patients with sickle cell disease have consistently reported an increased risk of mortality in patients with comparatively lower TRV thresholds $\geq 2.5$ $\mathrm{m} / \mathrm{s}^{7,8}$ The reasons behind such difference remain unclear. They may be attributed to observational study design and adjustment for cofounders, the underlying mechanism of $\mathrm{PH}$ (hemolysis vs. hypercoagulability), or the more severe anemia in patients with thalassemia which may contribute to TRV elevation beyond that attributed to $\mathrm{PH}$.

Although this study was not designed to evaluate the role of intervention, it does suggest a protective role for TRV improvements below the $3.2 \mathrm{~m} / \mathrm{s}$ threshold. There is a high unmet need for randomized clinical trials evaluating the role of intervention in $\beta$-thalassemia patients with $\mathrm{PH}$, to avoid the detrimental effects of this complication.

\section{Authors}

Giorgio Derchi, ${ }^{1 \star}$ Khaled M. Musallam, ${ }^{2 \star}$ Valeria Maria Pinto, ${ }^{3}$ Giovanna Graziadei, ${ }^{4}$ Marianna Giuditta, ${ }^{5}$ Susanna Barella, ${ }^{6}$ Raffaella Origa, ${ }^{6}$ Gavino Casu, ${ }^{7}$ Annamaria Pasanisi, ${ }^{8}$ Filomena Longo, ${ }^{9}$ Maddalena Casale, ${ }^{10}$ Roberta Miceli, ${ }^{11}$ Pierluigi Merella, ${ }^{7}$ Barbara Gianesin, ${ }^{12}$ Pietro Ameri, ${ }^{13,14}$ Immacolata Tartaglione, ${ }^{10}$ Silverio Perrotta, ${ }^{10}$ Antonio Piga, ${ }^{15}$ Maria Domenica Cappellini ${ }^{4,16}$ and Gian Luca Forni ${ }^{3}$ on behalf of the Webthal $\circledast$ project

1Department of Cardiology, High Specialty Ligurian Clinical Institute (ICLAS), Genoa, Italy; ${ }^{2}$ Thalassemia Center, Burjeel Medical City, Abu Dhabi, UAE; ${ }^{3}$ Center for Microcythemia, Congenital Anemia and Iron Dysmetabolism, Galliera Hospital, Genoa, Italy; ${ }^{4}$ Department of Medicine and Medical Specialties, IRCCS Ca' Granda Foundation, Maggiore Policlinico Hospital, Milan, Italy; ${ }^{5}$ Cardiovascular Disease Unit, IRCCS Ca' Granda Foundation, Maggiore Policlinico Hospital, Milan, Italy; ${ }^{6}$ Microcitemico Pediatric Hospital " Antonio Cao ", ARNAS G. Brotzu, Cagliari, Italy; ${ }^{7}$ Cardiology Unit, AO University of Sassari, Sassari, Italy; ${ }^{8}$ Hematology Unit, A. Perrino Hospital, Brindisi, Italy; ${ }^{9}$ Reference Centre for Hemoglobinopathies, AOU San Luigi Gonzaga Hospital, Orbassano, Italy; ${ }^{10}$ Department of Women, Child and General and Specialized Surgery, University Luigi Vanvitelli, Naples, Italy; ${ }^{11}$ Cardiology Unit, Galliera Hospital, Genoa, Italy; ${ }^{12}$ ForAnemia Foundation, Genoa, Italy; ${ }^{13}$ Cardiology Unit, IRCCS 
Ospedale Policlinico San Martino, Genoa, Italy; ${ }^{14}$ Department of Internal Medicine, University of Genoa, Genoa Italy; ${ }^{15}$ Department of Clinical and Biological Sciences, University of Turin, Turin, Italy and ${ }^{16}$ Department of Clinical Sciences and Community Health, University of Milan, Milan, Italy

${ }^{*} G D$ and KMM contributed equally as co-first authors.

Correspondence:

GIAN LUCA FORNI - gianluca.forni@galliera.it

https://doi.org/10.3324/haematol.2021.280389

Received: November 21, 2021.

Accepted: February 22, 2022.

Prepublished: March 3, 2022.

\section{Disclosures}

No conflicts of interest to disclose.

\section{Contributions}

GD, KMM, VMP and GLF conveived and designed the study; GD, VMP, GG, MG, SB, RO, GC, APa, FL, MC, RM, PM, BG, PA, IT, SP, APi, MDC and GLF collected data; KMM perfomed statistical analysis. All authors reviewed and interpreted the results. KMM drafted the manuscript. All authors reviewed the manuscript review for important intellectual content and approved the manuscript before submission.

\section{Data sharing statement}

Data can be made available upon request to the corresponding author.

\section{References}

1. Taher AT, Musallam KM, Cappellini MD. Beta-thalassemias. N Engl J Med. 2021;384(8):727-743.

2. Taher AT, Cappellini MD. How I manage medical complications of beta-thalassemia in adults. Blood. 2018;132(17):1781-1791.

3. Derchi G, Galanello R, Bina P, et al. Prevalence and risk factors for pulmonary arterial hypertension in a large group of betathalassemia patients using right heart catheterization: a Webthal study. Circulation. 2014;129(3):338-345.

4. Taher A, Musallam K, Cappellini MD. Guidelines for the Management of Non Transfusion Dependent Thalassaemia (NTDT). Vol 2. Nicosia, Cyprus 2017.

5. Pinto VM, Musallam KM, Derchi GE, et al. Mortality in betathalassemia patients with confirmed pulmonary arterial hypertension on right heart catheterization. Blood. 2022;139(13):2080-2083.

6. Morris CR, Kim HY, Trachtenberg F, et al. Risk factors and mortality associated with an elevated tricuspid regurgitant jet velocity measured by Doppler-echocardiography in thalassemia: a Thalassemia Clinical Research Network report. Blood. 2011;118(14):3794-3802.

7. Maitra P, Caughey M, Robinson L, et al. Risk factors for mortality in adult patients with sickle cell disease: a metaanalysis of studies in North America and Europe. Haematologica. 2017;102(4):626-636.

8. Gladwin MT, Sachdev V, Jison ML, et al. Pulmonary hypertension as a risk factor for death in patients with sickle cell disease. N Engl J Med. 2004;350(9):886-895. 